\title{
Meta-heuristic techniques for optimal design of analog and digital filter
}

\author{
El Beqal Asmae ${ }^{1}$, Kritele Loubna $^{2}$, Benhala Bachir ${ }^{3}$, Zorkani Izeddine $^{4}$ \\ ${ }^{1,2,4}$ Faculty of Sciences Dhar el Mahraz, University of Sidi Mohamed Ben Abdllah, Morroco \\ ${ }^{3}$ Faculty of Sciences, University of Moulay Ismail, Morroco
}

\begin{tabular}{|c|c|}
\hline Article Info & ABSTRACT \\
\hline Article history: & In this paper, two Meta-heuristic techniques; namely Ant Colony \\
\hline Received Jan 17, 2020 & Optimization (ACO) and Genetic Algorithm (GA) have been applied for \\
\hline Revised Feb 19, 2020 & used to solve multimodal optimization problem in Infinite Impulse Response \\
\hline Accepted Mar 8, 2020 & $\begin{array}{l}\text { (IIR) filter design and to select the optimal component values from industrial } \\
\text { series as well as to minimize the total design error of a } 2 \text { nd order Sallen-Key }\end{array}$ \\
\hline Keywords: & $\begin{array}{l}\text { active band-pass filter, also a comparison between the performances reached } \\
\text { by those two Meta-heuristics was made in this article. }\end{array}$ \\
\hline
\end{tabular}

$2^{\text {nd }}$ order Sallen-Key active

band-pass filter

Ant colony optimization

Genetic algorithm

IIR filter

Copyright () 2020 Institute of Advanced Engineering and Science.

Meta-heuristics

All rights reserved.

Optimization

Corresponding Author:

El Beqal Asmae,

Faculty of Sciences Dhar El Mhraz,

University of Sidi Mohamed Ben Abdllah, Fez, Morocco.

Email: asmae.elbekkal@gmail.com

\section{INTRODUCTION}

Filters are frequency selective circuits that allow the passage of certain frequencies and block the others. They can be either digital or analog. Digital filters use digital processors to perform mathematical calculations on the sampled values of the signal in order to perform the filter process. A computer or a dedicated Digital Signal Processor (DSP) could be used implementing digital filters. On the other hand, to perform the filtering operation, the analog filters use electronic components such as capacitors, resistors, operational amplifiers, transistors, etc. These types of filters are mainly used in the telecommunication field for noise reduction, signal enhancement, etc.

The conventional approach to filter design is to find the transfer function that satisfies the response specification, and to implement this transfer function in one of the standard circuit structures. In many cases, this approach is inadequate, and optimization is re-quired. The need for this optimization can arise in the case of computational complexity for digital filters and component selection in analog filters. Nowadays, Meta-heuristics based methods are widely used. Such techniques lead to solve real-world problems within a reasonable amount of time [1].

The complexity of IIR digital filter design lies to two major prob-lems, the first one is the filter can become unstable if the poles move outside the unit circle during the adaptation process and to deal with this problem, the parameter space is limited [2], the second issue is their error surface which is usually multimodal. In that case, conventional gradient-based methods fail to attain the global minimum and can easily converge towards local minima of error surface [3]. Consequently, an efficient search method based on a global search process must be used to overcome this problem. 
In the literature, various Meta-heuristic algorithms have been proposed to avoid IIR filter design problems. These algorithms include Genetic Algorithm (GA) [4], the Simulated Annealing (SA) [5], Particle Swarm Optimization (PSO) [6], Cat Swarm Optimization (CSO) [7], Differential Evolution Algorithm [2], Artificial Bee Colony (ABC) algorithm [8] and modified-interior search algorithm with Lèvy flight [9].

In the case of analog filters, to reduce costs and make the design faster, the selection of passive circuit components in active filter design is done from different manufactured series (E12, E24, E48, E96, and E192). To obtain the desired amplitude response, but this selection is difficult because of the number of possible filter combinations. In the literature, various Meta-heuristics were used for optimal design of analog circuits such as, Simulated Annealing (SA) [10], Genetic Algorithms (GA) [11], Tabu Search (TS) [12], Particle Swarm Optimization (PSO) [13], Ant Colony Optimization (ACO) [14-16] and Artificial Bee Colony (ABC) [17-18]. In this paper, we propose to apply two different Meta-heuristics, namely, the Ant Colony Optimization (ACO) and the Genetic Algorithm (GA), to solve the multimodal optimization problem in IIR filter design and for the optimal sizing of the analog filter considering two objectives functions, the midfrequency and the selectivity factor.

The remainder of the paper is structured as follows: The second section presents an overview of the ACO and GA techniques. The third section deals with the application of those techniques for the optimal design of analog and digital filter. The fourth section is devoted to the comparison of the performances of the two proposed techniques. Finally, the last section summarizes the main results of the work.

\section{ACO AND GA TECHNIQUES: AN OVERVIEW}

\subsection{The ant colony optimization}

ACO is a Meta-heuristic developed in order to solve difficult optimization problems. It has been inspired by the foraging behavior of real ant colonies. The ACO algorithm was introduced by Macro Dorigo and al. [19], and it was successfully applied to solve the traveling salesman problem (TSP) [20], vehicle routing problem [21], clustering [22]. For solving such problems, ants select the vertex to be visited based upon the random proportional rule $[19,23]$. Suppose ant $\mathrm{k}$ is in vertex $\mathrm{i}$, and then the probability of choosing the next vertex $\mathrm{j}$ is given by (1):

$$
P_{i j}^{k}= \begin{cases}\frac{\left(\tau_{i j}\right)^{\alpha} \cdot\left(\eta_{i j}\right)^{\beta}}{\sum_{l \in J_{i}^{k}}\left(\tau_{i j}\right)^{\alpha} \cdot\left(\eta_{i j}\right)^{\beta}} & \text { if } i \in J_{i}^{k} \\ O & \text { if } i \notin J_{i}^{k}\end{cases}
$$

Where $J_{i}^{k}$ is the set of neighbors of vertex $\mathrm{i}$ of the $\mathrm{K}^{\mathrm{th}}$ ant, $\tau_{\mathrm{ij}}$ is the amount of pheromone trail on edge (i,j), $\alpha$ and $\beta$ are parameters that determine the relative influence of the pheromone trails and the visibility value, i.e. $\eta_{\mathrm{ij}}$, which expression is given by (2):

$$
\eta_{i j}=\frac{1}{d_{i j}}
$$

The $d_{i j}$ is the distance between vertices $\mathrm{i}$ and $\mathrm{j}$.

After all ants have completed a tour, the pheromone trails are up-dated. The update follows this rule:

$$
\tau_{\mathrm{ij}}=(1-\rho) \cdot \tau_{\mathrm{ij}}+\sum_{\mathrm{k}=1}^{\mathrm{m}} \Delta \tau_{\mathrm{ij}}^{\mathrm{k}}
$$

where $\rho$ is the evaporation rate, $\mathrm{m}$ is the number of ants, and $\Delta \tau_{\mathrm{ij}}^{\mathrm{k}}(\mathrm{t})$ is the quantity of pheromone laid on edge $(I, j)$ by ant: 


$$
\Delta \tau_{i j}^{k}=\left\{\begin{array}{l}
\frac{Q}{L_{k}} \text { if ant } k \text { used edge }(i, j) \text { in its tour } \\
0 \quad \text { otherwise }
\end{array}\right.
$$

$Q$ is a constant and $L_{k}$ is the length of the tour constructed by ant k.

As shown in Figure 1 the procedure of the ACO technique through the following flowchart:

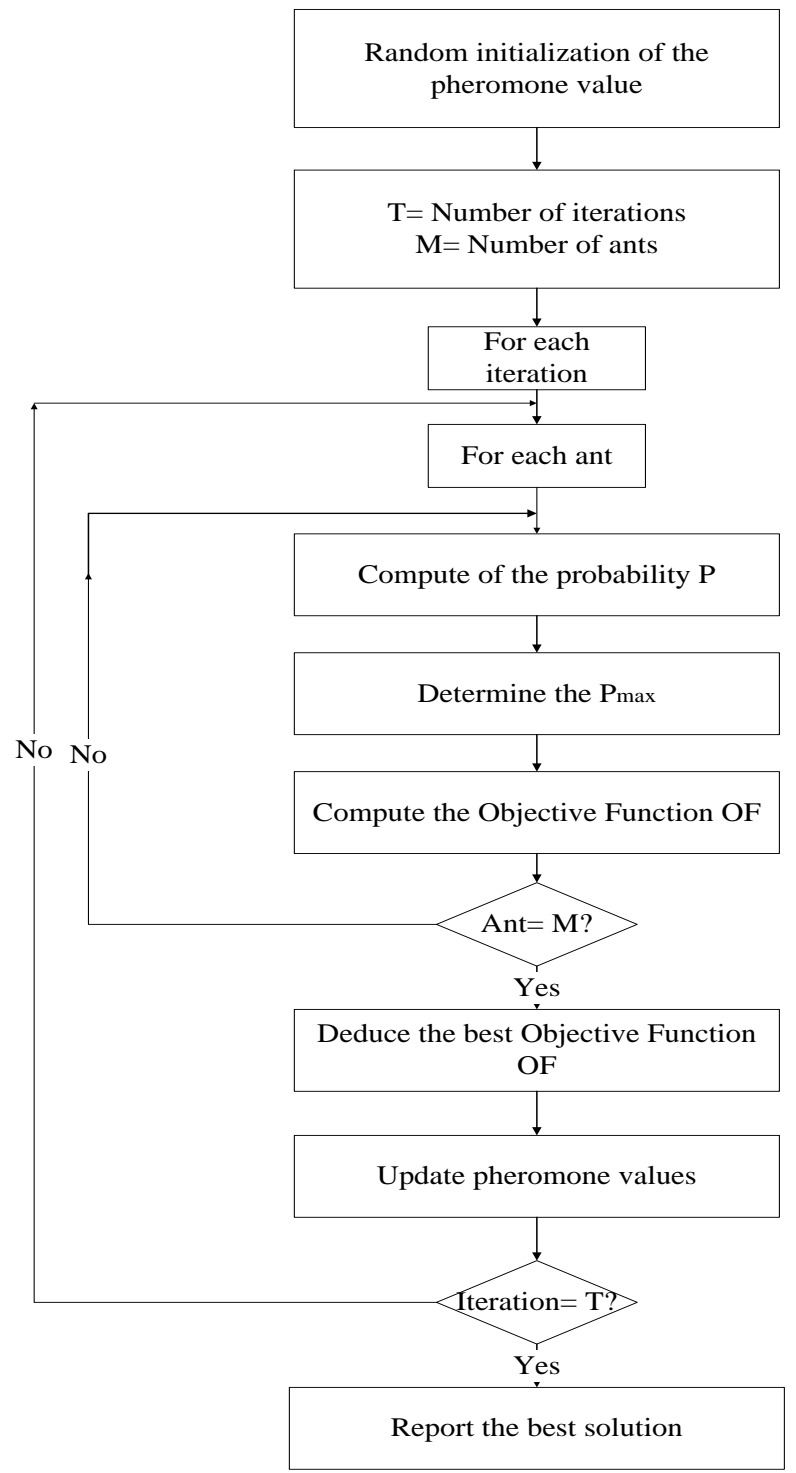

Figure 1. Flowchart of the ACO technique

\subsection{The genetic algorithm}

The GA finds their origins in the biological processes of survival and adaptation. Its principle consists of sampling a population of potential solutions. A population of individuals is, initially, randomly generated. The GA performs then operations of selection, crossover and mutation on the individuals, corresponding respectively to the principal of survival of the fittest, recombination of genetic material and random mutation observed in nature [24]. The optimization process is carried out through the generation of successive populations until a stop criterion is met.

The flowchart in Figure 2 provides an overview of a GA procedure [24]. 


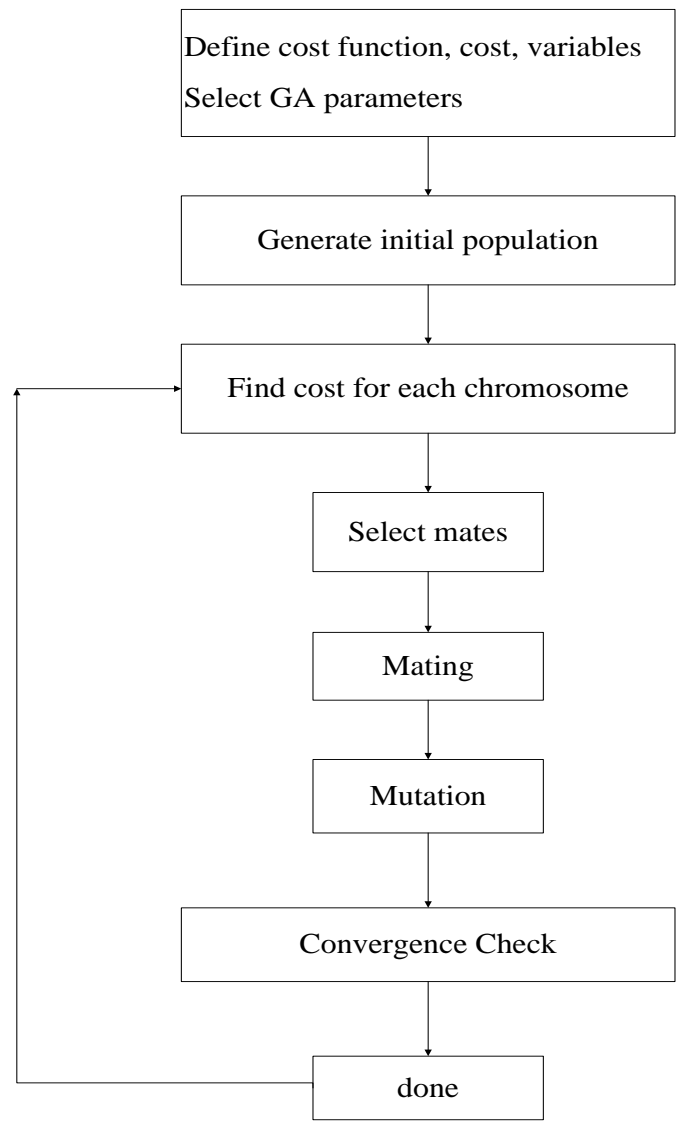

Figure 2. Flowchart of the GA

There are therefore 6 elements necessary for the running of the GA [24]:

1. We begin the process of fitting the problem to a GA by defining a chromosome as an array of variable values to be optimized.

2. The user must fix a priori the sizing parameters of the algorithm, in particular, the size of the population and the number of generations (which is very often used as a condition for stopping the algorithm).

3. Then the Generation of the initial population (set of possible solutions) can be random or from known approximate solution(s).

4. Each chromosome has a cost found by evaluating the cost function $\mathrm{f}$ at the variables. The higher this cost, the greater is the chance of an individual (solution) being selected for reproduction.

5. Now is the time to decide which chromosomes in the initial population are fit enough to survive and possibly reproduce offspring in the next generation, the costs and associated chromosomes are ranked from lowest cost to highest cost. The rest die off.

6. Then recombination/reproduction is achieved through two genetic operators, namely crossover and mutation.

a) The crossover combines (mates) two chromosomes (parents) to produce a new chromosome (offspring). The idea behind crossover is that the new chromosome may be better than both of the parents if it takes the best characteristics from each of the parents.

b) Mutation is usually considered as an auxiliary operator to extend the search space and causes release from a local optimum when used cautiously with the selection and crossover systems.

Operations of selection, crossover, and mutation are repeated until a favorable number of individuals for the new generation are created, and the objective function is calculated again for all of the individuals in the new generation. The best individual in the new generation according to its fitness is kept to continue to the next generation. Thus, the fitness of the entire population will be decreased with the reproduction of the generation. 


\section{APPLICATION TO THE ANALOG AND DIGITAL FILTER DESIGN}

\subsection{The analog filter: second order sallen-key active band-pass filter}

The Sallen-Key circuit has the advantage that the quality factor $(Q)$ can be varied via the inner gain $(G)$ without modifying the mid-frequency $\left(f_{m}\right)$. A drawback is, however, that $(Q)$ and the gain $\left(A_{m}\right)$ at the mid-frequency $\left(f_{m}\right)$ cannot be adjusted independently [25]. A circuit diagram for second-order Sallen-Key band-pass filter is shown in Figure 3.

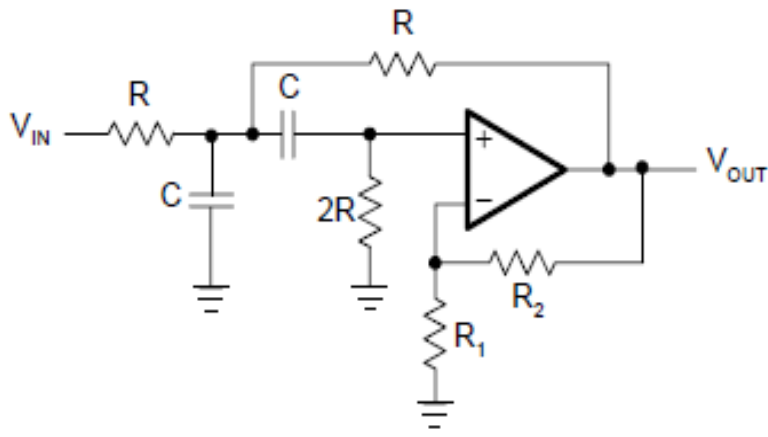

Figure 3. Schematic diagram of $2^{\text {nd }}$ order Sallen-Key active band-pass filter

The general transfer function for a second-order band-pass filter is:

$$
\mathrm{A}(\mathrm{s})=\frac{\frac{\mathrm{A}_{\mathrm{m}}}{\mathrm{Q}} \mathrm{s}}{1+\frac{1}{\mathrm{Q}} \mathrm{s}+\mathrm{s}^{2}}
$$

The Sallen-Key bandpass circuit in Figure 3 has the following transfer function:

$$
\mathrm{A}(\mathrm{s})=\frac{\mathrm{GRC} \omega_{\mathrm{m}} \mathrm{s}}{1+\left[\mathrm{RC} \omega_{\mathrm{m}}(3-\mathrm{G})_{\mathrm{s}}\right]+\left(\mathrm{R}^{2} \mathrm{C}^{2} \omega_{\mathrm{m}}^{2} \mathrm{~s}^{2}\right)}
$$

Through coefficient comparison with (5), obtain the following equations:

Mid-frequency: $\quad \mathrm{f}_{\mathrm{m}}=\frac{1}{2 \pi \mathrm{RC}}$ or $\omega_{\mathrm{m}}=\frac{1}{\mathrm{RC}}$

Inner gain:

$$
\mathrm{G}=1+\frac{\mathrm{R}_{2}}{\mathrm{R}_{1}}
$$

Gain at $\mathrm{f}_{\mathrm{m}}$ :

$$
A_{m}=\frac{G}{3-G}
$$

Filter quality:

$$
\mathrm{Q}=\frac{1}{3-\mathrm{G}}
$$

The mid-frequency $\left(\omega_{\mathrm{m}}\right)$ and the selectivity factor $(\mathrm{Q})$ of the filter, depend only on the values of the passives components, the specifications for the desired bandpass filter are $\omega_{\mathrm{m}}=6.28 \mathrm{k} \mathrm{rad} / \mathrm{s}$ $\left(\mathrm{f}_{\mathrm{m}}=1 \mathrm{KHz}\right)$ and $\mathrm{Q}=10$.

In order to generate $\omega_{\mathrm{SK}}$ and $\mathrm{Q}_{\mathrm{SK}}$ approaching the specified values; the values of the resistors and capacitors should be carefully selected. For this, we define the Total-Error $\left(\mathrm{TE}_{\mathrm{SK}}\right)$ which expresses the offset values, of the mid frequency and the selectivity factor, compared to the desired values, by:

$\mathrm{TE}_{\mathrm{SK}}=0.5 \Delta \omega_{\mathrm{SK}}+0.5 \Delta \mathrm{Q}_{\mathrm{SK}}$ 
Where:

$$
\Delta \omega_{\mathrm{SK}}=\frac{\left|\omega_{\mathrm{SK}}-\omega_{\mathrm{m}}\right|}{\omega_{\mathrm{m}}} \quad \Delta Q_{S K}=\frac{\left|\mathrm{Q}_{\mathrm{SK}}-\mathrm{Q}\right|}{Q}
$$

The aim is to obtain the exact values of design parameters (R, R1, R2 and C) which equate the TotalError $_{\mathrm{SK}}\left(\mathrm{TE}_{\mathrm{SK}}\right)$ to a very close value to 0 . The decision variables are the resistors and capacitors forming the circuit. Each component must have a value of the standard series (E12, E24, E48, E96, and E192).

\subsection{Digital filter: IIR filter}

IIR filters have been highly preferable in real world applications; namely, in system identification since an IIR filter can meet the desired performance levels. Therefore, in the simulation study, one adopts a system identification perspective to the IIR filters design. The principal task in system identification in this study is to vary the coefficients of the adaptive IIR filter iteratively using ACO and GA algorithms unless and until the filter's output signal is matched to the output signal of the unknown system when the same input signal is applied simultaneously to both the adaptive filter and unknown system under consideration. The Basic block diagram for system identification using an adaptive IIR filter is shown in Figure 4.

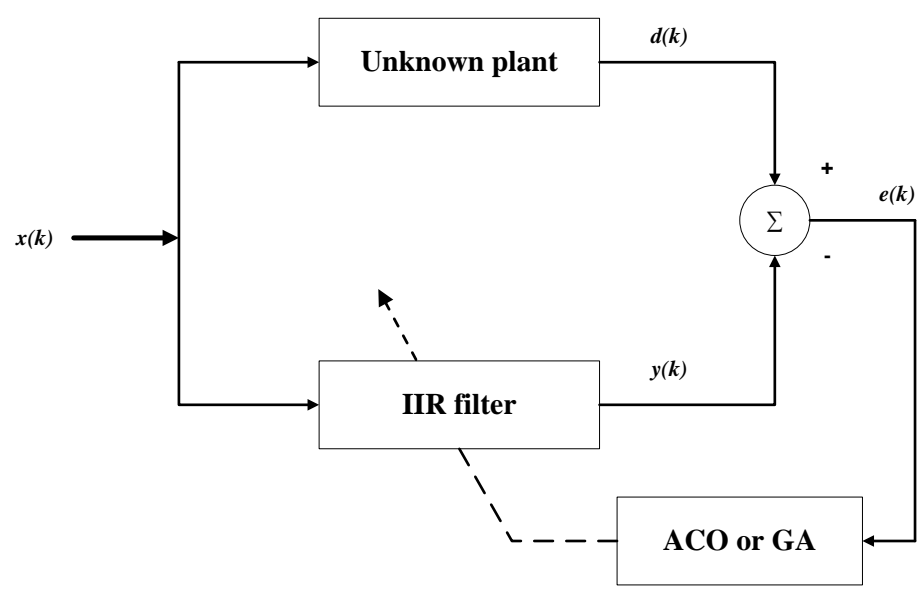

Figure 4. Block diagram of system identification

Consider the IIR filter with the input-output relationship given by [2, 8]:

$$
y(k)+\sum_{i=1}^{M} a_{i} y(k-i)=\sum_{i=0}^{L} b_{i} x(k-i)
$$

Where $\mathrm{x}(\mathrm{k})$ and $\mathrm{y}(\mathrm{k})$ are the input and the output of the filter, respectively, $\mathrm{M}$ is the filter's order, $(i=1,2, \ldots, M)$ and $(i=0,1, \ldots, L)$ are the filter coefficients. The transfer function of this IIR filter can be written as follows:

$$
H(z)=\frac{B(z)}{A(z)}=\frac{\sum_{i=0}^{L} b_{i} z^{i}}{1+\sum_{i=1}^{M} a_{i} z^{i}}
$$

Then an IIR filter design can be considered as an optimization problem with the Mean Square Error (MSE) between the output of the unknown system and the designed filter. The objective function is expressed as follow [2]:

$$
J(w)=\frac{1}{N} \sum_{k=1}^{N}(d(k)-y(k))^{2}
$$


where:

$\mathrm{N}$ is the number of input samples to be used. The design goal is to minimize the objective function $\mathrm{J}(w)$ by adjusting the filter coefficient vector $w$ represented as: $w=\left[b_{0} b_{1} \ldots b_{L} a_{0} a_{1} \ldots a_{M}\right]^{T}$

\section{RESULTS AND COMPARISON}

In order to carry out the comparison of the algorithms in similar conditions, the values of similar control parameters of the algorithms were chosen to be equal to each other; for example, population size and generation number rate. Table 1 shows the control parameter values used for the two algorithms in digital and analog filters design.

Table 1. The ACO and GA parameters

\begin{tabular}{|c|c|c|c|c|}
\hline \multirow{2}{*}{ Parameters } & \multicolumn{2}{|r|}{ Analog filter } & \multicolumn{2}{|r|}{ Digital filter } \\
\hline & $\mathrm{ACO}$ & GA & $\mathrm{ACO}$ & GA \\
\hline Population size & 800 & 800 & 200 & 200 \\
\hline Iteration cycles & 1000 & 1000 & 500 & 500 \\
\hline Evaporation rate $(\rho)$ & 0.1 & - & 0.1 & - \\
\hline Quantity of deposit (Q) & 0.4 & - & 0.4 & - \\
\hline Pheromone Factor $(\alpha)$ & 1 & - & 1 & - \\
\hline Heuristics Factor $(\beta)$ & 1 & - & 1 & - \\
\hline $\mathrm{q}_{0}$ & 0.2 & - & 0.2 & - \\
\hline Crossover & - & Two Point Crossover & - & Two Point Crossover \\
\hline Mutation rate & - & 0.0001 & - & 0.001 \\
\hline Selection probability & - & $50 \%$ & - & $50 \%$ \\
\hline
\end{tabular}

\subsection{Second order sallen-key active band-pass filter result}

The optimal values, linear and those following the different series of resistors and capacitors forming the studied filter and the performance associated with these values using the ACO and the GA techniques are shown in the following Tables 2-3.

Table 2. Values of the components and related filter performance for the ACO

\begin{tabular}{ccccccc}
\hline & Linear Values & E12 & E24 & E48 & E96 & E192 \\
\hline $\mathrm{R} 1(\mathrm{~K} \Omega)$ & 25.20 & 27.00 & 24.00 & 24.90 & 24.90 & 25.20 \\
$\mathrm{R} 2(\mathrm{~K} \Omega)$ & 47.88 & 47.00 & 47.00 & 48.70 & 47.50 & 48.01 \\
$\mathrm{R}(\mathrm{K} \Omega)$ & 45.50 & 47.00 & 47.00 & 46.40 & 45.64 & 45.30 \\
$\mathrm{C}(\mathrm{nF})$ & 3.50 & 3.30 & 3.60 & 3.48 & 3.48 & 3.48 \\
$\Delta \omega_{\text {SK }}$ & 0.00009 & 0.0267 & 0.0589 & 0.2571 & 0.0026 & 0.0101 \\
$\Delta \mathrm{Q}_{\mathrm{SK}}$ & 0.00000 & 0.6143 & 1.4000 & 1.2636 & 0.0826 & 0.0544 \\
$\mathrm{TE}_{\text {SK }}$ & 0.000045 & 0.3205 & 0.7294 & 0.7603 & 0.0426 & 0.0322 \\
\hline
\end{tabular}

Table 3. Values of the components and related filter performance for the GA

\begin{tabular}{ccccccc}
\hline & Linear Values & E12 & E24 & E48 & E96 & E192 \\
\hline $\mathrm{R} 1(\mathrm{~K} \Omega)$ & 24.809 & 27.00 & 24.90 & 24.90 & 24.90 & 24.90 \\
$\mathrm{R} 2(\mathrm{~K} \Omega)$ & 47.138 & 47.00 & 47.00 & 46.40 & 47.50 & 47.00 \\
$\mathrm{R}(\mathrm{K} \Omega)$ & 02.618 & 02.70 & 02.61 & 02.61 & 02.61 & 02.61 \\
$\mathrm{C}(\mathrm{nF})$ & 60.82 & 56.00 & 62.00 & 61.09 & 60.40 & 61.20 \\
$\Delta \omega_{\mathrm{SK}}$ & $5.6 \times 10^{-5}$ & 0.0531 & 0.0488 & 0.0144 & 0.0101 & 0.0031 \\
$\Delta \mathrm{Q}_{\mathrm{SK}}$ & 0.00036 & 0.6143 & 1.4000 & 0.2676 & 0.0826 & 0.1107 \\
$\mathrm{TE}$ & 0.00021 & 0.3337 & 0.7244 & 0.1410 & 0.0464 & 0.0569 \\
\hline
\end{tabular}

According to the results, we notice that the ACO algorithm achieved the smallest design error than GA for linear values and for E192 series. The following Figure 5 shows the PSPICE simulation of the filter gain for the second order Sallen-Key active band-pass filter. The practical mid frequency using the ACO and GA is equal to $1 \mathrm{KHz}$. Table 4 shows the comparison between the theoretical values and those practices for the error on the mid frequency for the optimal results; we notice that the practical value is equal to 0 for both algorithms, and that proved the validity of the proposed techniques. 


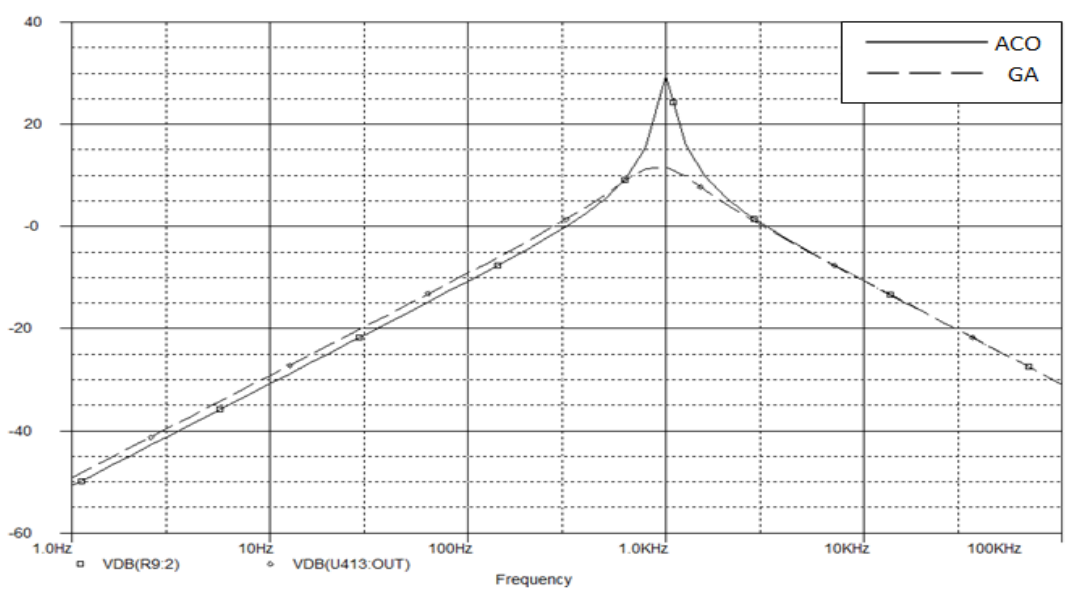

Figure 5. Frequency responses of second order Sallen-Key active band-pass Filter for the ACO and GA

Table 4. Comparisons between the theoretical and practices for the error on the mid-frequency

\begin{tabular}{ccc}
\hline & $\Delta \omega$ & $\Delta \omega$ \\
& theoretical & Practical \\
\hline ACO & 0.0101 & 0.0000 \\
GA & 0.0031 & 0.0000 \\
\hline
\end{tabular}

\subsection{IIR filter result}

In this section, three examples are used in the simulation studies. ACO and GA algorithms are used for the digital filter design; the performance of the two proposed algorithms was also compared.

For the three examples, the input $\mathrm{x}(\mathrm{k})$ was a uniform white sequence of data samples length $\mathrm{N}=50$.

Exemple 1: This example is taken from [2, 7-8]. A second order system was considered, and the filter was a first order IIR filter. Hence, the transfer functions are given by:

$$
H_{s}(z)=\frac{0.05-0.4 z^{-1}}{1-1.1314 \mathrm{z}^{-1}+0.25 \mathrm{z}^{-2}}, H(z)=\frac{b}{1-a z^{-1}}
$$

Exemple 2: In this example, a fifth order system was considered and the filter was a forth order IIR filter [7]. Hence, the transfer functions are given by:

$$
\begin{aligned}
& H_{s}(z)=\frac{0.1084+0.5419 \mathrm{z}^{-1}+1.0837 \mathrm{z}^{-2}+1.0837 \mathrm{z}^{-3}+0.5419 \mathrm{z}^{-4}+0.1084 \mathrm{z}^{-5}}{1+0.9853 \mathrm{z}^{-1}+0.9738 \mathrm{z}^{-2}+0.3864 \mathrm{z}^{-3}+0.1112 \mathrm{z}^{-4}+0.0113 \mathrm{z}^{-5}} \\
& H(z)=\frac{b_{0}+b_{1} z^{-1}+b_{2} z^{-2}+b_{3} z^{-3}+b_{4} z^{-4}}{1-a_{1} z^{-1}-a_{2} z^{-2}-a_{3} z^{-3}-a_{4} z^{-4}}
\end{aligned}
$$

Exemple 3: This example is taken from [7]. The 3rd order system shown in (18) is modeled using a 2nd order filter. Hence the transfer function of the model is expressed in (19).

$$
\begin{aligned}
& H_{S}(z)=\frac{-0.2-0.4 z^{-1}+0.5 z^{-2}}{1-0.6 z^{-1}+0.25 z^{-2}-0.2 z^{-3}} \\
& H(z)=\frac{a_{0}+a_{1} z^{-1}}{1-b_{1} z^{-1}-b_{2} z^{-2}}
\end{aligned}
$$

For the three examples, the search space is limited between -2 and 2. The best, the worst, the average and the standard deviation (SD) of the MSE values of the ACO and GA algorithms over 30 runs are presented in Table 5. From this table we can notice that for the first example not only the best and mean 
values, even the worst MSE values yielded by GA are the lowest, as compared to those of ACO algorithm, for the two other examples, the smaller MSE and SD is achieved by using the ACO, so it is obvious that when the order of the filter and the system increase, the ACO become much better than GA.

The parameter values of the filters designed with the best MSE values using the proposed algorithms are given in Table 6. The MSE values versus the number of iterations are plotted in Figures 6-8 for the examples 1, 2 and 3 respectively. From these figures, we can notice that the GA converges to the minimum values faster than the ACO. However, the ACO algorithm achieves the smaller MSE values for examples 2 and 3 .

Table 5. Performance measures for the

\begin{tabular}{cccc}
\multicolumn{4}{c}{ four examples } \\
\hline Examples & MSE & ACO & GA \\
\hline & Best & 0.0144 & $2.16 \times 10^{-6}$ \\
Example 1 & Worst & 0.0282 & $2.21 \times 10^{-5}$ \\
& Average & 0.0205 & $8.46 \times 10^{-6}$ \\
& SD & 0.0033 & $5.32 \times 10^{-6}$ \\
\hline & Best & $4.0 \times 10^{-5}$ & 0.0002 \\
Example 2 & Worst & $6.48 \times 10^{-4}$ & 0.1270 \\
& Average & $2.33 \times 10^{-4}$ & 0.0076 \\
& SD & $1.59 \times 10^{-4}$ & 0.0232 \\
\hline & Best & $4.3 \times 10^{-4}$ & 0.0019 \\
Example 3 & Worst & $1.80 \times 10^{-3}$ & 0.0040 \\
& Average & $1.04 \times 10^{-3}$ & 0.0028 \\
& SD & $3.27 \times 10^{-4}$ & 0.0005 \\
\hline
\end{tabular}

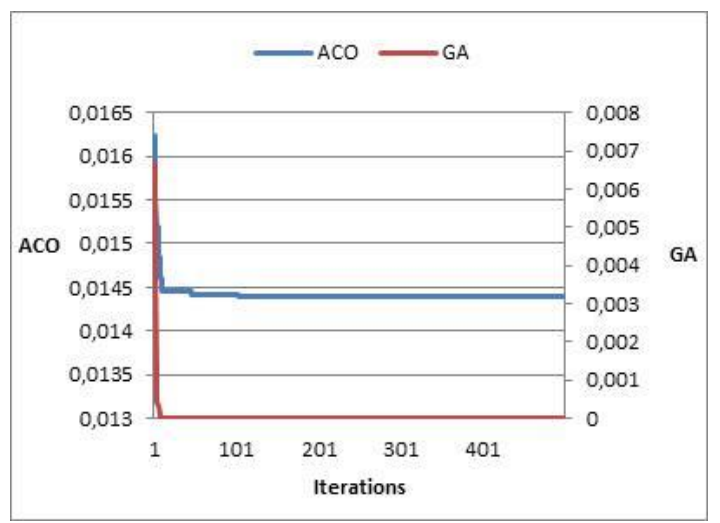

Figure 6. MSE values versus the number of iteration for ACO and GA algorithms (Example 1)
Table 6. Optimized parameters of the filters designed with the best MSE found using ACO and GA

\begin{tabular}{cccc}
\hline Examples & coefficients & ACO & GA \\
\hline \multirow{2}{*}{ Example 1 } & $a$ & 0.892 & 0.218 \\
& $b$ & -0.335 & 0.050 \\
\hline \multirow{5}{*}{ Example 2 } & $a_{1}$ & -0.776 & 0.246 \\
& $a_{2}$ & -0.749 & -0.445 \\
& $a_{3}$ & -0.206 & 0.265 \\
& $a_{4}$ & -0.041 & -0.062 \\
& $b_{0}$ & 0.106 & 0.106 \\
& $b_{1}$ & 0.520 & 0.413 \\
& $b_{2}$ & 0.976 & 0.494 \\
& $b_{3}$ & 0.859 & 0.101 \\
Example 3 & $b_{4}$ & 0.325 & -0.135 \\
\hline & $a_{0}$ & -0.227 & -0.205 \\
& $a_{1}$ & -0.599 & -0.582 \\
& $b_{1}$ & -0.143 & -0.185 \\
& $b_{2}$ & -0.374 & -0.372 \\
\hline
\end{tabular}

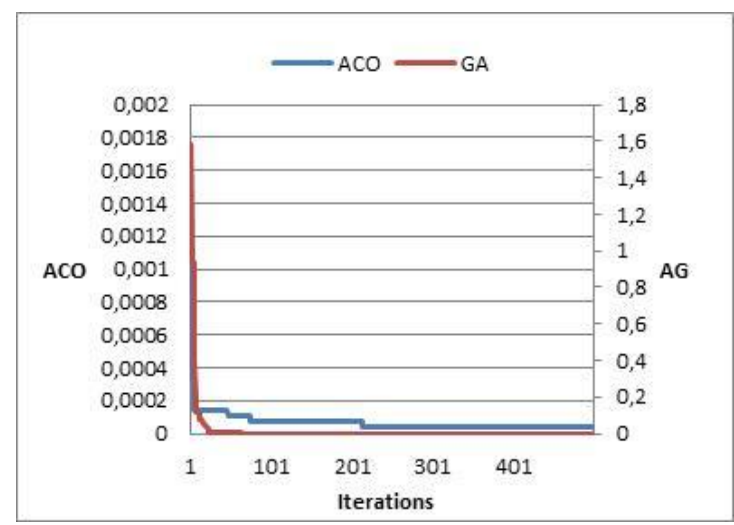

Figure 7. MSE values versus the number of iteration for ACO and GA algorithms (Example 2)

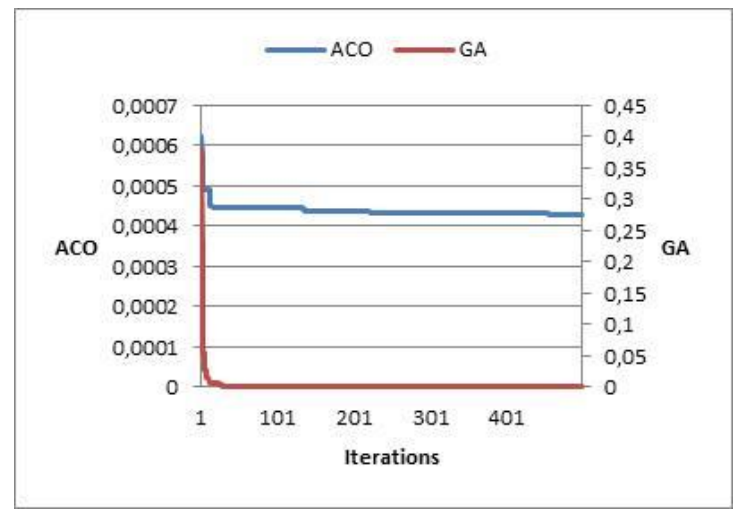

Figure 8. MSE values versus the number of iteration for ACO and GA algorithms (Example 3) 
Figure 9 presents the pole-zero plots for the example 2 obtained with the filter parameters presented in Table 6 using the ACO (a) and GA techniques (b), the position of poles within the unit circle shows that the designed filters are stable. Table 7 gives a comparison between the MSE values achieved by the proposed algorithms and those from the literature, this comparison reveals that the ACO provides the smallest MSE value for example 2 and 3, and GA gives the minimum MSE for the first example.

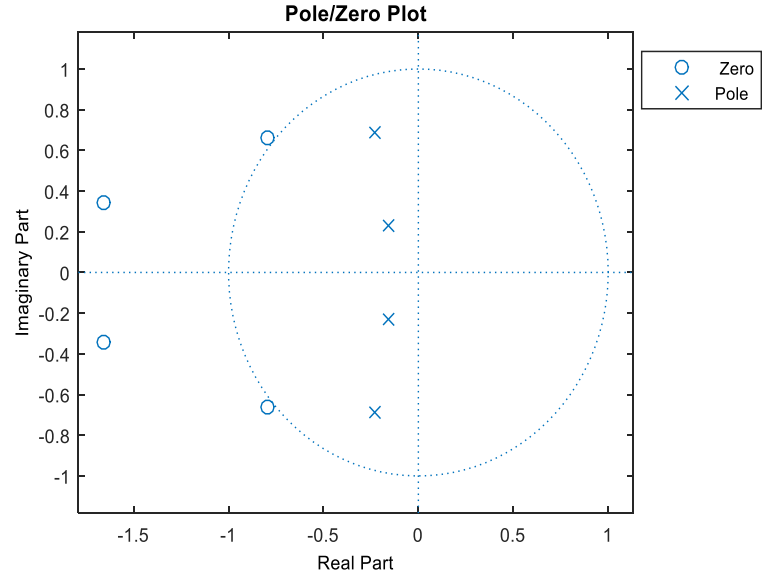

(a)

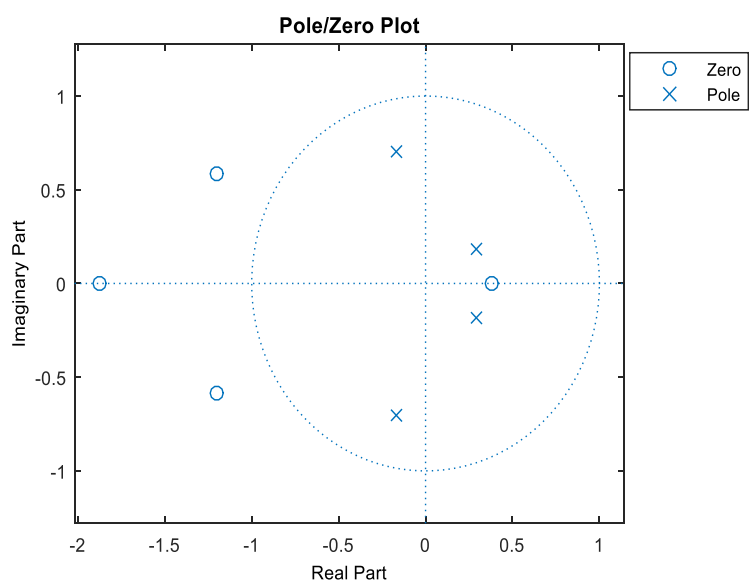

(b)

Figure 9. Pole-zero plot for the filter designed by ACO algorithm (a) and by GA (b) using coefficients presented in Table 6 (example 2)

Table 7. Performance comparison of different reported MSE values

\begin{tabular}{cccc}
\hline Examples & Reference & Proposed algorithm & MSE Value \\
\hline \multirow{4}{*}{ Example 1 } & Panda and al.[7] & CSO & 0.0175154 \\
& Karaboga [8] & PSO & 0.0646 \\
& Present work & ACO & 0.0144 \\
& Panda and al.[7] & GA & $2.16 \times 10^{-6}$ \\
\hline \multirow{2}{*}{ Example 2 } & & CSO & $6.9475 \times 10^{-5}$ \\
& Present work & PSO & $6.93727 \times 10^{-5}$ \\
& Panda and al.[7] & GCO & $4.0 \times 10^{-5}$ \\
& G. Upadhyay and al.[26] & CSO & 0.0002 \\
\hline \multirow{2}{*}{ Example 3 } & FFA & 0.00139 \\
& Present work & ACO & $4.3 \times 10^{-4}$ \\
& & GA & 0.0019 \\
\hline
\end{tabular}

\section{CONCLUSION}

In this paper, we have presented an application of the Ant Colony Optimization technique and the Genetic Algorithm for the optimal design of an analog filter : the second order Sallen-Key active bandpass filter, and a digital filter : IIR filter. For the analog filter we selected the optimal values of discrete components from different manufactured series. The optimal sizing of the analog filters with high accuracy is successfully achieved, and the validity of the proposed techniques was proved via PSPICE simulations. For the IIR filter, the design results have confirmed the effectiveness of the proposed methods to handle unknown system identification problem, GA for reduced order filter and ACO for the higher one. The stability of the designed filters has been proved by the position of the poles using optimal coefficients. Then, the comparative study between the ACO algorithm and the GA affirmed that the ACO technique is better than the GA in term of the accuracy of results since the ACO has achieved the smallest values of the error design and the MSE.

\section{REFERENCES}

[1] Ibrahim H, Osman, and J.P. Kelly, "Meta-heuristics: theory and applications," Kluwers Academic Publishers, Boston, 1996. 
[2] N. Karaboga, "Digital IIR filter design using differential evolution algorithm," EURASIP Journal on Applied Signal Processing, vol. 8, no. 8, pp. 1269-1276, 2005.

[3] S.C.NG, et al., "The Genetic Search Approach a New Learning Algorithm for Adaptative IIR Filtring," IEEE Signal Processing Magazine, pp. 38-46, 1996.

[4] K.S. Tang, et al., "Design and optimization of IIR filter structure using hierarchal genetic algorithms," IEEE Transactions on Industrial Electronics, vol. 45, no. 3, pp. 481-487, 1998.

[5] S. Chen, et al., "Digital IIR filter design using adaptive simulated annealing," Digital Signal Processing, vol. 11, no. 3, pp. 241-251, 2001.

[6] B. Luitel and G.K. Venayagamoorthy, "Particle swarm optimization with quantum infusion for system identification," Engineering Applications of Artificial Intelligence, vol. 23, pp. 635-649, 2010.

[7] G. Panda, et al., "IIR system identification using cat swarm optimization," Expert Systems with Applications, vol. 38, pp. 12671-12683, 2011.

[8] N. Karaboga, "A new design method based on artificial bee colony algorithm for digital IIR filters, " Journal of the Franklin Institute, vol. 346, no 4, pp. 328-348, 2009.

[9] M.Kumar, et al., "Adaptive infinite impulse response system identification using modified-interior search algorithm with Lèvy flight," ISA Transactions, vol. 67, pp. 266-279, 2017.

[10] J. Dreo, et al., "Metaheuristics for hard optimization: Methods and case studies," New York: Springer, 2006.

[11] A. El beqal, et al., " Sizing of three-stage bipolar transistor amplifier by the genetic algorithm," 4th International Conference on Optimization and Applications (ICOA'18), 2018.

[12] F. Glover, "Tabu search-part I," ORSA Journal on computing, vol. 1, no. 3, pp. 190-206, 1989.

[13] F. T. S. Chan and M. K. Tiwari, "Swarm Intelligence: focus on ant and particle swarm optimization," I-Tech Education and Publishing, 2007.

[14] B. Benhala, "An improved aco algorithm for the analog circuits design optimization," International Journal of Circuits, Systems and Signal Processing, ISSN: 1998-4464, vol. 10, pp.128-133, 2016.

[15] L. Kritele, et al., "Ant Colony Optimization for Optimal Low-Pass State Variable Filter Sizing," International Journal of Electrical and Computer Engineering (IJECE), vol 8, no 1, pp. 227-235, 2018.

[16] L. Kritele, et al., "Optimal Digital IIR Filter Design Using Ant Colony Optimization," IEEE 4th International Conference on Optimization and Applications (ICOA'18), pp. 1-5, 2018.

[17] H. Bouyghf, et al., " Analysis of the impact of metal thickness and geometric parameters on the quality factor-Q in integrated spiral inductors by means of artificial bee colony technique," International Journal of Electrical and Computer Engineering (IJECE), vol. 9, no 4, pp. 2918-2931, 2019.

[18] H. Bouyghf, et al., "Optimal design of RF CMOS circuits by means of an artificial bee colony technique," Chapter 11, Book: Focus on swarm intelligence research and applications, Eds., B. Benhala, P. Pereira and A. Sallem, NOVA Science Publishers, pp. 221-246, 2017.

[19] M. Dorigo and S. Krzysztof, "An Introduction to Ant Colony Optimization," a chapter in Approximation Algorithms and Metaheuristics, a book edited by T. F. Gonzalez, 2006.

[20] Y. Jinhui, et al., "An ant colony optimization method for generalized TSP problem," Progress in Natural Science, vol. 18, no. 11, pp. 1417-1422, 2008.

[21] Q. Chengming, "Vehicle Routing Optimization in Logistics Distribution Using Hybrid Ant Colony Algorithm," TELKOMNIKA Indonesian Journal of Electrical Engineering, vol 11, no 9, pp. 5308-5315, 2013.

[22] A. S. Girsang, et al., "Fast Ant Colony Optimization for Clustering," Indonesian Journal of Electrical Engineering and Computer Science, vol. 12, no. 1, pp. 78-86, 2018.

[23] M. Dorigo, et al., "Ant algorithms for discrete optimization," Artificial Life Journal, vol. 5, pp. 137-172, 1999.

[24] R.L. Haupt and S.E. Haupt, "Practical GeneticAlgorithms," (book) John Wiley \& Sons 2004, ISBN 0-471-45565-2.

[25] Ron Mancini, "Op Amps for Everyone Design Reference," 2nd Edition, Elsevier, 2003.

[26] P. Upadhyay, et al., " A new design method based on firefly algorithm for IIR system identification problem," Journal of King Saud University - Engineering Sciences, vol. 28, pp. 174-198, July 2016. 